\title{
Copper Nanoparticles by Laser Ablation Confined in Methanol in the Presence of an Argon Gas Environment
}

\author{
David Omar Oseguera Galindo* and Eden Oceguera Contreras** \\ * Instituto Tecnológico José Mario Molina Pasquel y Henríquez, Unidad Académica Tamazula, \\ Tecnológico Nacional de México, Tamazula de Gordiano, 49650, Jalisco, México \\ E-mail: david.omar0927@hotmail.com \\ ${ }^{*}$ Centro de Nanociencias y Nanotecnología, Universidad Nacional Autónoma de México, Ensenada, Baja \\ California, 22860, México \\ ${ }^{* *}$ Centro Universitario de los Valles, Universidad de Guadalajara, Ameca, 46600, Jalisco, México
}

\begin{abstract}
The fundamental emission of Nd:YAG laser $(1064 \mathrm{~nm})$, with an energy $0.1 \mathrm{~J} / \mathrm{pulse}$ and a repetition rate of $10 \mathrm{~Hz}$ was employed to synthesize nanoparticles with the laser ablation method confined in methanol. Furthermore in the synthesis was used an experimental design that allow injection Argon gas to prevent combustion. The sample showed an absorption peak characteristic of copper nanoparticles. The optical properties of nanoparticles were analyzed by comparing the experimentally absorption with the extinction efficiency of the Mie Theory, including in the theoretical calculations a size distribution obtained through in the micrographs, resulting in both spectrum present an absorption peak $\approx 570 \mathrm{~nm}$. Meanwhile, in these nanoparticles was used the Energy dispersive x-ray spectroscopy (EDS) showing high purity of the copper element.
\end{abstract}

DOI: 10.2961/jlmn.2017.03.0021

Keywords: copper nanoparticles, laser ablation, flammable solvent, absorbance

\section{Introduction}

Metallic nanoparticles are being very interesting in different disciplines due to their optical and electronic properties that present in size nano scale. One of the main characteristics of these nanoparticles is an absorption peak in the visible spectra which is extensively related to the superficial plasmon resonance. Specially, copper nanoparticles show an absorption peak around $570 \mathrm{~nm}$, their are increasing the attention as application in nanofluids, lubrication, catalysis, light emitting diodes and because they are significantly lower cost than gold and silver [1, 2].

The laser ablation technique is an alternative method reported for the first time in 1993 and since then, the number of articles published of this method is increasing [3]. This is because, the method offers certain advantages, such as the short time for the synthesis and it is not necessary use reagents that may contaminate their surface. This happen, thanks to generation of the plasma due to high power laser interaction on a solid target immersed in a liquid medium. The plasma is constituted for ions, atoms and electrons of the solid material confined for the high density of the medium, while electronic recombination is attribute to the rapid cooling, causing the ions to pass to their neutral state, favoring the nanoparticles formation [4]. However to obtain copper nanoparticles, the solvent plays an important role in the synthesis, for instance, employing the laser Nd:YAG on copper solid immersed in ethanol and acetone, using UV-Vis spectroscopy, both samples display an absorption peak near to $590 \mathrm{~nm}$, whereas in distilled water this peak is absent, indicating oxidized nanoparticles [5]. On the other hand, there is an article reporting high quality of copper nanoparticles oxide-free with the molar concentration of polyvinylpyrrolidone (PVP) in distilled water. To analysis the purity of this sample, they used diffraction X- ray technique where nanoparticles obtained in PVP showed copper crystalline phases [6].

The aim of this work was to obtain copper nanoparticles by laser ablation method in a safe environment and analyzes the purity of these nanoparticles. The sample prepared in methanol was characterized with UV-Vis spectroscopy and Transmission electron microscopy. In addition, to get knowledge of the purity of nanoparticles was used the Mie Theory and energy dispersive x-ray spectral analysis technique

\section{Experimental}

We employed the fundamental emission of Nd:YAG laser $(1064 \mathrm{~nm})$, with an energy $0.1 \mathrm{~J} /$ pulse, laser fluence of $12.7 \mathrm{~J} / \mathrm{cm}^{2}$, a repetition rate of $10 \mathrm{~Hz}$ and used the experimental design reported by Oseguera et al. in the synthesis, where they prepared silver nanoparticles in inflammable solvents [7]. They show a design to allow inject a flux of high purity Argon gas to purging Oxygen to from the vessel in order to prevent combustion ignition. In this case, we employed a copper solid as a target, during the interaction with the laser irradiation that was $10 \mathrm{~min}$, the target was kept fix and it was immersed in $15 \mathrm{ml}$ of methanol and distilled water. It should be mentioned, in distilled water it was not used Argon gas.

The samples in both solvents were characterized by UVVisible absorption spectroscopy with a Perkin-Elmer 330 spectrophotometer. However, only in methanol the nano- 
particles were prepared on nickel grid for observation by the transmission electron microscopy JEOLJEM-2100F and Energy dispersive x-ray spectroscopy (EDS). In addition, the size distribution of nanoparticles were obtained through the analysis of micrographs

\section{Results and discussion}

Figure 1 shows absorption spectra of nanoparticles suspension in distilled water and methanol. We can mentioned some differences in the spectra, in methanol present the absorption peak around $570 \mathrm{~nm}$, which is related to the localized surface plasmonic resonance of copper nanoparticles nearly spherical, this according with the Mie theory where the peak absorption shift depend of particles size and of the optical properties of the solvent [8]. However, in distilled water the absorption peak is absent, it has the appearance of oxidized nanoparticles $\left(\mathrm{CuO}\right.$ or $\left.\mathrm{Cu}_{2} \mathrm{O}\right)$, indicating that oxidation of copper took place due to presence of oxygen in water, as has been reported elsewhere [9, 10].
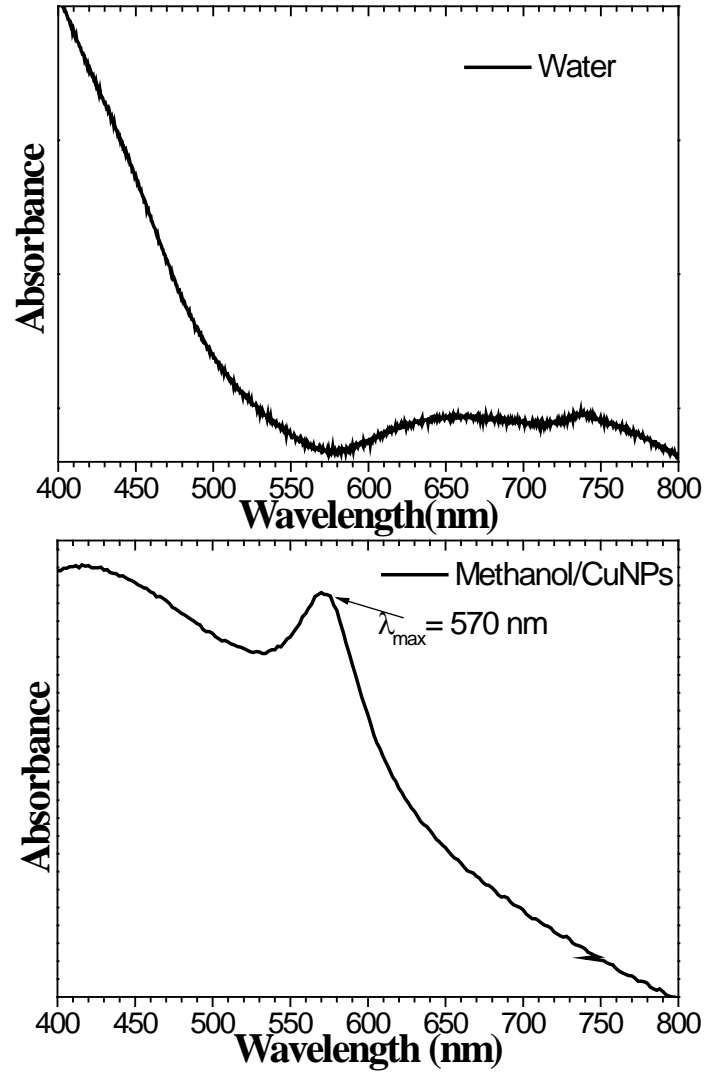

Fig. 1 UV-Vis absorbance spectra for nanoparticles prepared in water (up) and methanol (down).

Figure 2 presents a micrograph of nanoparticles obtained in methanol, as observe, they tend to agglomerate and the shape that they show is quasi spherical, regarding the geometry there is some correlation with the absorption peak of nanoparticles suspended in this solvent. In the figure 3 is presented the corresponding size distribution, the histogram was fitted with Gaussian curves, where the main peak is centered around $22 \mathrm{~nm}$ while the other is centered to a big- ger size $54 \mathrm{~nm}$, central position of these peaks as well as their standard deviation is show in the figure, resulting a wide size distribution.

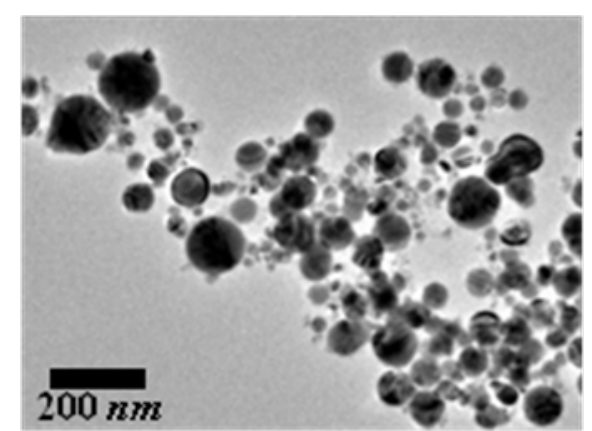

Fig. 2 Transmission electron micrographs nanoparticles obtained by the fundamental emission (1064 nm).

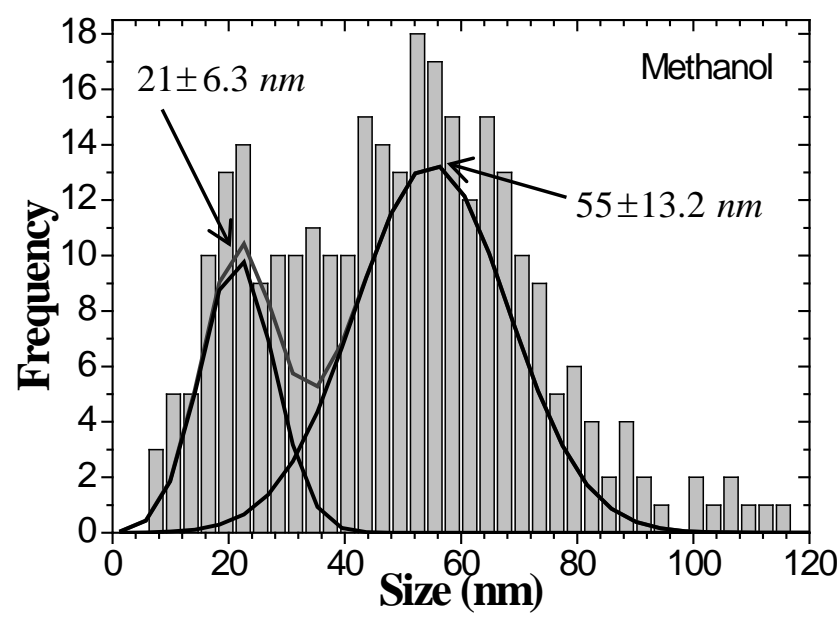

Fig. 3 Size distributions of nanoparticles prepared in methanol.

To analyze optical properties of $\mathrm{Cu}$ nanoparticles obtained in methanol from data of UV-Vis spectrum, we used the Mie theory for the calculated numerical of the extinction efficiency due to the relation of this function have with the experimentally absorption [11]. In this case, in the FORTRAN code was assumed the refractive index of methanol, whereas the complex refractive index of a bulk $\mathrm{Cu}$ was taken from the experimental work of Jhonson and Chistry et al 1972 [12]. In addition, we calculated the weighted mean of extinction efficiency, which take account the histogram of the size distributions (figure 3), so that we can represented it by

$$
Q_{e x t}=\frac{\sum n\left(r_{i}\right) Q_{e x t}\left(r_{i}\right)}{N}
$$

Where $Q_{\text {ext }}$ is the extinction efficiency of a spherical particle of radius $r, n\left(r_{i}\right)$ is the particles number with the same radius and $N$ is the total number particles of the size distribution. In the figure 4 , is compare the absorption experimental spectrum of nanoparticles in methanol with the theoretical extinction efficiency, resulting that in both spec- 
tra the absorption peak is around $570 \mathrm{~nm}$ and have similar appearance in the range of $400-500 \mathrm{~nm}$. Nevertheless, the experimentally absorption from $600 \mathrm{~nm}$ is biggest; it is possible that agglomeration of nanoparticles as a showed in the micrographs must be included this effect in the FORTRAN code to better the theoretical spectrum adjustment. On the other hand, this spectral comparison suggests that nanoparticles obtained in methanol have certain purity of $\mathrm{Cu}$.

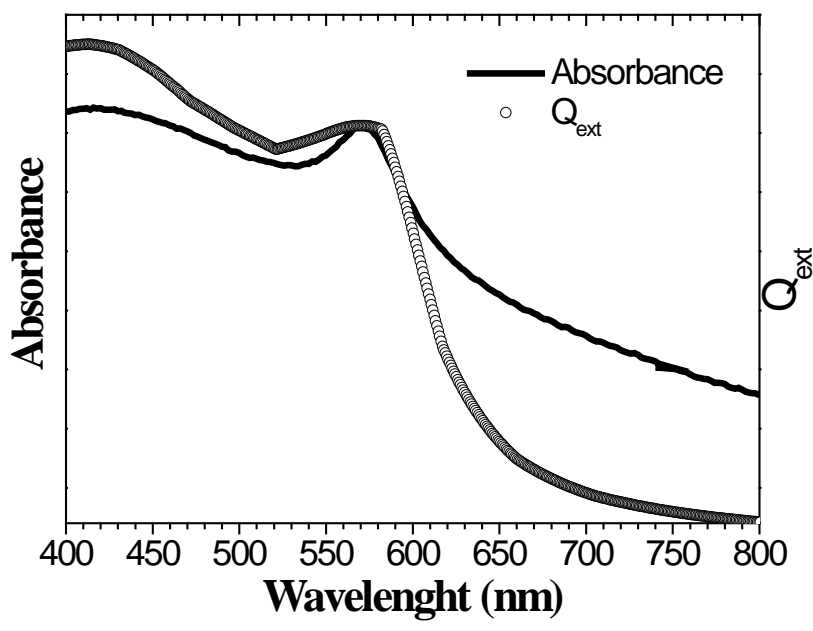

Fig. 4 Comparison the experimentally absorbance of nanoparticles in methanol with the calculated extinction efficiency given a size distribution.

Respect to the analysis the purity of nanoparticles; they were characterized by energy dispersive X-ray spectroscopy (EDS). Figure 5, present all elements analyzed and inside is the image where EDS was done. According to the result, a strong $k$-alpha peak about $8 \mathrm{KeV}$ confirm the presence of $\mathrm{Cu}$, meanwhile the second peak was due to Ni grid. It is worth mentioned that we analyzed EDS in other micrographs and similar results was found, indicating the nanoparticles were oxygen free

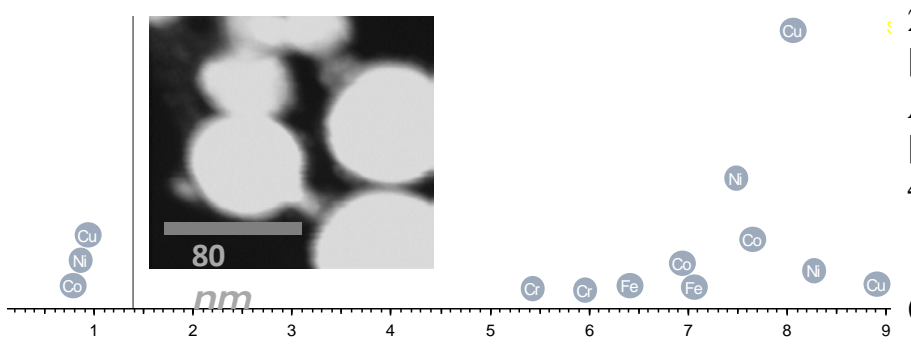

Fig. 5 EDS spectrum of nanoparticles obtained in methanol ing this useful to acquire better knowledge of optical properties of this kind of materials. Furthermore, employing the energy dispersive $\mathrm{X}$-ray spectroscopy, $\mathrm{Cu}$ was the element analyzed in nanoparticles. So that, we can conclude had successfully obtained copper nanoparticles in methanol, using laser ablation technique in an argon gas environment.

\section{Acknowledgments}

Authors want to thanks the financial support from Consejo Nacional de Ciencia y Tecnología (CONACYT), Ingeniería en Innovación Agrícola Sustentable of ITJMMPyH Unidad Académica Tamazula. Also, we want to thank the Centro de Nanociencias y Nanotecnología (CNYN-UNAM) for the space and facilities to realize the research. In addition, authors deeply thank the technical assistance of Francisco Ruiz Medina and Jaime Mendoza (CNYN-UNAM).

\section{References}

[1] T. M. D. Dang, T.T.T. Le, E. Fribourg-Blanc and M.C. Dang: Adv. Nat. Sci: Nanosci. Nanotechnol., 2, (2011) 025004.

[2] D. Dorranian, S. A. A. Afshar, N. Tahmasebi, and A. F. Eskandari: J. Cluster Sci., 4, (2014) 1147.

[3] A Fojtik, A Henglein, Ber. Buns. Phys. Chem 97, (1993) 252

[4] D. O. Oseguera Galindo, I.C Andrade, A.M. Benítez, G.G. Rosas, A Chávez Chávez, A. Pérez Centeno and M. A Santana Aranda: J. Mater. Sci. Eng B., 4, (2014) 279.

[5] P.V. Kazakevich, A. V. Simakin, V.V. Voronov, and G.A. Shafeev: Appl. Surf. Sci., 252, (2006) 4373.

[6] K.D. Malviya, and K.Chattopadhyay: J. Mater. Sci., 50, (2015), 980.

[7] D.O Oseguera Galindo, O. Hernández Utrera, R. Machorro Mejía and M. A. Santana Aranda: J. Laser Micro/Nanoengin., 11, (2016) 158.

[8] C.F Borhen and D.R Huffman, Absorption and Scattering of Light by Small Particles ( Wiley, New York, 1983)

[9] A. Nath, and A. Khare: J. Appl. Phys., 110, (2011) 043111.

[10] M.A. Gondal, T.F. Qahtan, M.A. Dastageer, T.A. Saleh, Y. W. Maganda, and D.H. Anjum: Appl. Surf. Sci., 286, (2013) 149.

[11] W. Haiss, N.T. Thanh, J. Aveyard, and D.G Fernig:

Anal. Chem., 79, (2007) 4215.

[12] P. B. Johnson, R.W. Christy: Phys. Rev. B., 6, (1972) 4370.

(Received: April 7, 2017, Accepted: September 3, 2017)

\section{Conclusions}

Our result suggest, some correlation between the experimentally absorption with the extinction efficiency with a given size distribution, resulting that in both spectra present the absorption peak characteristic of $\mathrm{Cu}$ nanoparticles, be- 